\section{The Social Determinants of Health: An Imperative for Canadian Public Health}

A 15 -year-old boy living in Lesotho has about a 10\% chance of reaching the age of 60 years, whereas a 15-year-old Swede has a $91 \%$ chance. Sir Michael Marmot often uses this example when discussing the work of the WHO Commission on the Social Determinants of Health which began its work in 2005 and is due to submit its final report in 2008. One does not need to go to foreign countries to get a sense of the importance of social and economic factors in health. A stroll through most of our Canadian cities and towns will do as well. For example, the life expectancy at birth for men born in the low-income Montreal neighbourhood of the «CLSC des Faubourgs» is 67.8 years compared to 78.4 years for those born in the contiguous territory of the "CLSC Metro" neighbourhood just a few streets to the West. ${ }^{1}$ Indeed, there are many examples throughout the country that clearly show that health inequalities are alive and well in Canada.

Although Canada has in many ways been central to the development of the concepts of population health, it has to a great extent been less than successful in applying its lessons in its own backyard. The Lalonde Report, the Ottawa Charter for Health Promotion, and more recently the work of the Canadian Institute for Advanced Research all contributed substantially to redefining the principles of public health and social interventions to promote a just and healthy society for all citizens. In this vein, the upcoming CPHA annual conference (Halifax, June 1-4) is focused on the social determinants of health. The Conference theme "Reducing Health Inequalities through Evidence and Action" was chosen to take stock of the upcoming WHO report and the large body of scientific evidence, and to set the stage for actions to promote health equity within the country and abroad.

Environmental interventions have always been central to public health, from Hippocrates' On Airs, Waters, and Places to John Snow removing the handle of the Broad Street pump. Today, the social environment, including social and economic inequities, are at the root of many diseases worldwide. The Canadian public health community must be at the forefront of the interventions and research required to deal with this challenge. Removing that handle will seem like child's play compared to the tasks ahead, but we, like Snow, cannot shy away from our collective responsibility.

\section{Gilles Paradis}

Interim Scientific Editor

\section{Reference}

1. CLSC des Faubourgs and CLSC Metro, 1997-2001 data, obtained from the Carrefour montréalais d'information sociosanitaire, Agence de la santé et des services sociaux de Montréal. Available online at: http://www.cmis.mtl.rtss.qc.ca/fr/atlas/etat_sante_pop/details_sante_ globale.html\#top (Accessed February 21, 2008).
LE MOT DE LA RÉDACTION

\section{Les déterminants sociaux de la santé : un devoir pour la santé publique canadienne}

Pour un garçon de 15 ans vivant au Lesotho, la probabilité d'atteindre l'âge de 60 ans est d'environ $10 \%$, tandis que pour un Suédois de 15 ans, elle est de $91 \%$. Sir Michael Marmot emploie souvent cet exemple lorsqu'il parle de la Commission des déterminants sociaux de la santé de l'OMS, qui a commencé ses travaux en 2005 et devrait déposer son rapport final en 2008. Mais il n'est pas nécessaire d'aller à l'étranger pour se faire une idée de l'importance des facteurs sociaux et économiques pour la santé. Une balade dans la plupart des villes et localités du Canada fait très bien l'affaire. Par exemple, l'espérance de vie à la naissance des hommes nés dans un quartier à faible revenu de Montréal, le CLSC des Faubourgs, est de 67,8 ans, contre 78,4 ans pour les natifs d'un quartier adjacent, CLSC Métro, quelques rues plus à l'ouest ${ }^{1}$. À vrai dire, on trouve dans tout le pays de nombreux exemples qui montrent clairement que les inégalités en santé se portent bien au Canada.

Même si à bien des égards le Canada a joué un rôle central dans l'élaboration des concepts de la santé des populations, il a été considérablement moins fort pour en appliquer les leçons dans sa propre cour. Le rapport Lalonde, la Charte d'Ottawa pour la promotion de la santé et, plus récemment, les travaux de l'Institut canadien des recherches avancées ont beaucoup contribué à redéfinir les principes d'intervention en matière sociosanitaire afin de favoriser une société juste et saine pour tous les citoyens. Dans le même ordre d'idées, la prochaine conférence annuelle de l'ACSP (Halifax, $1^{\text {er }}$ au 4 juin) portera sur les déterminants sociaux de la santé. Le thème choisi, "Vers une réduction des inégalités en santé par la recherche et l'action ", vise à faire le point sur le rapport de l'OMS à paraître et sur les multiples preuves scientifiques et à préparer la voie à des mesures de promotion de l'équité en santé au pays et à l'étranger.

Les interventions sur le milieu ont toujours été au coeur de la santé publique, du traité Des airs, des eaux et des lieux d'Hippocrate à l'initiative de John Snow, qui en ôtant le levier d'une pompe à eau d'un quartier de Londres a endigué une épidémie de choléra. Aujourd'hui, le milieu social, y compris les inégalités socioéconomiques, est à l'origine de nombreuses maladies dans le monde. Les intervenants canadiens en santé publique doivent être aux premières lignes de la pratique et de la recherche pour relever ce défi. Ôter un simple levier peut sembler facile comparé à la tâche qui nous attend, mais comme Snow, nous avons une responsabilité collective devant laquelle nous ne pouvons pas détourner le regard.

Le rédacteur-réviseur scientifique intérimaire,

Gilles Paradis

\section{Référence}

1. CLSC des Faubourgs et CLSC Métro, données de 1997-2001 obtenues auprès du Carrefour montréalais d'information sociosanitaire, Agence de la santé et des services sociaux de Montréal. Sur Internet : http://www.cmis.mtl.rtss.qc.ca/fr/atlas/ etat_sante_pop/details_sante_globale.html\#top (consulté le 21 février 2008). 